\title{
Microsurgical Management of Vestibular Schwannomas with Brainstem Compression: Surgical Challenges and Outcome
}

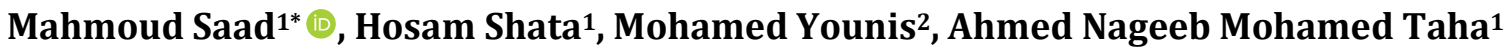 \\ ${ }^{1}$ Department of Neurosurgery, Faculty of Medicine, Mansoura University, Mansoura, Egypt \\ ${ }^{2}$ Department of Anesthesiology, Faculty of Medicine, Mansoura University, Mansoura, Egypt \\ Email: ${ }^{\star d r} \_$mhmodsaad@yahoo.com
}

How to cite this paper: Saad, M., Shata, H., Younis, M. and Taha, A.N.M. (2020) Microsurgical Management of Vestibular Schwannomas with Brainstem Compression: Surgical Challenges and Outcome. Open Journal of Modern Neurosurgery, 10, 122-134.

https://doi.org/10.4236/ojmn.2020.101013

Received: November 29, 2019

Accepted: December 17, 2019

Published: December 20, 2019

Copyright $\odot 2020$ by author(s) and Scientific Research Publishing Inc. This work is licensed under the Creative Commons Attribution International License (CC BY 4.0).

http://creativecommons.org/licenses/by/4.0/

(c) (i) Open Access

\begin{abstract}
Background: Vestibular schwannomas exerting brainstem compression with or without hydrocephalus are challenging for neurosurgeons regarding the plan of surgery, operative risk and postoperative sequale. Purpose: To report our experience with the surgical challenges and outcomes for Hannover type T4a \& T4b vestibular schwannomas. Methods: Retrospective case study conducted at neurosurgery department, Mansoura University between October 2016 and May 2019 of 16 patients with vestibular schwannoma compressing the brainstem. We reviewed their clinical, radiological, surgical data, incidence of complications and outcome. Results: We had 16 patients: 10 of $\mathrm{Ha}-$ nover grade T4b and 6 of grade T4a. Gross total resection was achieved in 2 cases while subtotal resection was achieved in 5 cases and in the remaining 9 cases; only partial resection was feasible. We had 4 cases of mortality. Among new onset morbidity after surgery, 3 cases had post-operative bulbar manifestation and 4 cases had brainstem dysfunction. Conclusion: Vestibular schwannomas compressing the brain stem are challenging in their clinical presentation, surgery and post-operative outcome. The outcome of patients treated with hydrocephalus prior to vestibular schwannoma surgery is better than those who did initial VS surgery. Patients presented with significant brainstem, bulbar manifestation had worse outcome and needed longer ICU stay and longer time for recovery.
\end{abstract}

\section{Keywords}

Brainstem, Challenges, Vestibular Schwannoma

\section{Introduction}

Among the tumors of the cerebellopontine angle (CPA), vestibular schwannoma 
(VS) is the most common tumor. It is a benign, slow-growing neoplasm. It can reach into large tumors [1] [2] [3].

Otological complaints are the early presenting symptoms which may be neglected by the patients, till it reaches progressively a large size; then present by severe clinical presentations; cerebellar, brainstem, cranial nerve compression in posterior fossa (oculomotor nerve, trochlear nerve, trigeminal nerve, abducens and lower cranial nerves), and obstructive hydrocephalus. Size and extension of VS is a determinator of treatment plan, clinical, surgical outcome and prognosis. As tumor reaches a huge size, it increases the potentiality of postoperative facial nerve dysfunction and hearing loss with high incidence of hazardous morbidity and mortality. However, it remains a great challenge, because the neurosurgeons are total resection of tumors, saving the neurological functions, reducing the incidence of perioperative severe complications and achieving better long-term survival and better quality of life [4] [5] [6].

The goals of surgical treatment of VS include radical excision of tumor with facial nerve and hearing function preservation and avoidance of severe perioperative complications with the main concern in preserving patients' vital and functional maximum safety as surgery, because giant VS carries significant higher exposure of the patient to vital risks, hazardous complications, and functional disabilities [7] [8] [9] [10] [11].

Radical tumor resection in a single session if possible is the standard treatment of choice in the management of VS. Difficulties in achieving that goal created other options as staged resection or radiosurgery following partial resection. Surgical management of giant VS is a unique challenging mission for neurosurgeons; it has abundant blood supply, difficult clear identification of facial nerve and cochlear nerve to avoid their dysfunction during surgery and surgical handling of such large tumors can lead to dysfunction of cerebellum and brainstem resulting in postoperative major complications [12] [13] [14].

The aim of our retrospective study is to analyze clinical presentation, microsurgical management, and outcomes of VS with brainstem compression with (T4b) and without (T4a) radiological signs of fourth ventricle compression or shift according to the Hannover grading scale.

\section{Patients and Methods}

There were 54 patients with VS treated at our institution between October 2016 and May 2019; out of them 16 cases of tumors exerting brain stem compression (Hannover type T4a "without hydrocephalus", T4b "with hydrocephalus") (Table 1) were selected to be included in this retrospective study conducted at Neurosurgery Department, Mansoura University after approval obtained from local institutional review board committee.

Retrospective analysis of patients' medical record (clinical status and postoperative complications), follow-up data collected throughout follow-up period and radiology were done. Patients' data included: size of the tumor size, extent 
of resection, surgical and clinical outcome including short-and long-term complications were reviewed and analyzed.

Preoperative radiological evaluation included: High-resolution bone window computed tomography (CT) brain studies, for assessment of anatomy of posterior semicircular canal, the posterior wall of the internal auditory canal, a high jugular bulb and radiologic signs of hydrocephalus. All patients underwent contrast enhanced MRI brain for evaluation of tumor boundaries, relations and size.

Preoperative and postoperative facial nerve function was assessed using House-Brackmann (H-B) grading scale [15]. We categorized facial nerve function into 3 categories: good (H-B I + II), fair (H-B III), and poor (H-B IV + V + VI). Audiological examination for assessment of hearing function was done using pure tone audiometry (PTA) and speech discrimination score (SDS). The preoperative and postoperative hearing assessment was determined by guidelines of world health organization for grading of hearing loss (Table 2) [16].

Tumors were assessed regarding size, consistency, cerebellar and brainstem compression, presence of arachnoid plane and associated hydrocephalus using was post-contrast $\mathrm{CT}$ scan and magnetic resonance imaging (MRI) brain study. Postoperatively; all patients were subjected to early follow-up CT scan of the brain (done within first 24 hours) to evaluate postoperative sequelae as postoperative surgical bed hematomas or hydrocephalus, then follow-up MRI at 3 months interval after the surgery for assessment of residual tumor, and then yearly for assessment of tumor recurrence.

All cases were operated either through retrosigmoid approach via the park bench position in 10 cases and via sitting position in 6 patients. The choice of position was based on surgeon preference rather than tumor characteristics. Intraoperative monitoring (electromyography of facial nerve and auditory evoked

Table 1. Hannover VS tumor extension grading scale [1].

\begin{tabular}{cc}
\hline Grade & Tumor \\
T1 & Purely intracanalicular \\
T2 & Intrameatal or extrameatal \\
T3a & Filling the cerebellopontine cistern \\
T3b & Reaching the brainstem \\
T4a & Compression of the brainstem \\
T4b & Compression of the brainstem with dislocation of the fourth ventricle \\
\hline
\end{tabular}

Table 2. World health organization classification of hearing loss grading.

\begin{tabular}{cc}
\hline Grades of hearing loss & Hearing level in decibel \\
\hline Mild & $20-40 \mathrm{~dB}$ \\
Moderate & $41-60 \mathrm{~dB}$ \\
Sever & $61-80 \mathrm{~dB}$ \\
Profound & $>80 \mathrm{~dB}$ \\
\hline
\end{tabular}


potentials) was feasible only in 6 cases during the surgery. All the patients were admitted immediately postoperative in the Neurocritical Care Unit (ICU); the length of ICU stay was evaluated and correlated with the surgical outcome.

The patients' data were gathered, coded and analyzed using IBM SPSS Statistics Program (Version 21) for Windows. Continuous variables' data are presented as median (range) and categorical Variables data are presented as number (percent). Quantitative variables as age were reported as mean \pm standard deviation. Qualitative variables as sex distribution and underlying disease were reported as proportion (percentage).

\section{Results}

Out of 16 patients, 9 are males (56.25\%), 7 females (43.75\%). The range of age at presentation 40 - 68 years, the mean age was 55.7. All of 16 patients were complaining of variable degrees of hearing deficit; profound hearing loss had reported in 3 cases (18.75\%), moderate hearing loss had occurred in 4 patients (25\%) and mild diminution of hearing in 9 patients (56.25\%). Preoperatively; 9 patients $(56.25 \%)$ had bulbar manifestation due to lower cranial nerves compression; 2 patients out of them needed preoperative tracheostomy for the severity of their bulbar manifestation. 7 patients (43.75\%) suffered long tracts signs and hemiparesis (brainstem dysfunction). 6 patients (37.5\%) had ataxic gait (cerebellar manifestation). Four patients (25\%) had preoperative facial pain due to trigeminal nerve compression. 6 patients (37.5\%) suffered facial nerve affection; 2 patients had hemifacial spasm and 4 patients suffered moderate palsy (HB Grade II-III) (Table 3).

Ten patients $(62.5 \%)$ had $4^{\text {th }}$ ventricular shift associated with preoperative hydrocephalus; 3 cases were operated upon by implantation of ventriculoperitoneal

Table 3. Patients demographics and clinical presentation.

\begin{tabular}{cc}
\hline Patients & No. (\%) \\
\hline Gender & $9(56.25 \%)$ \\
Male & $7(43.75 \%)$ \\
Female & \\
Age & $40-68$ years \\
Range & 55.7 years \\
Average & \\
Clinical presentations & \\
Hearing loss & Profound 3 (18.75\%), moderate $4(25 \%)$, mild $9(56.25 \%)$ \\
Lower CN (bulbar manifestation) & $9(56.25 \%$ \\
Brainstem (long tract sings) & $7(43.25 \%)$ \\
Cerebellar manifestation & $6(37.5 \%)$ \\
Facial dysfunction & $4(25 \%)$ \\
Trigeminal dysfunction & $6(37.5 \%)$ \\
Hydrocephalus & $10(62.5 \%)$ \\
\hline
\end{tabular}


shunt for acute hydrocephalic manifestation; 2 of them were admitted in the emergency hospital with disturbed conscious level related to the hydrocephalus. In one case; the shunt was done electively for severe headache and papilloedema on fundus examination.

In 3 cases ( 2 cases T4b and 1 case T4a); the shunt was done after surgery. The first case the patient did not recovered well after the surgery with partial resection of the tumor. Immediate postoperative CT showed progressive hydrocephalus due to small hematoma in the tumor bed and blood in the subarachnoid space. In the second case the procedure was aborted because of tense cerebellum and occurrence of significant hemorrhage during tumor dissection and 3 days after surgery the patient developed severe headache and vomiting and follow up CT showed progression of preoperative hydrocephalic changes and she was shunted. In the third case the tumor (T4a) was partially removed and 2 months after surgery the patient developed severe headache and vomiting and progressive dysequilibrium and CT revealed hydrocephalus and was shunted.

The surgical plan was determined by careful assessment of the neurological and medical status of the patients based on the Karnofsky performance scale (KPS) and anaesthetic evaluation using the American Society of Anaesthesiology (ASA) physical status classification system done by consultant neuroanaesthesiologist [17] (Table 4).

Based on functional, general condition evaluation through KPS \& ASA scores; older patients more than $60 \mathrm{yrs}$. $(n=7,43.75 \%) .5$ patients ( 3 patients T4b and 2 patients T4a) had poor scores (KPS $\leq 70$, ASA score 3, 4). All of them were planned for parial intracpsular tumor resection to decompress the brainstem and in one of them shunt was implanted second day after surgery due to progressive hydrocephalus. 2 patients older than 60 years ( 1 patient T4b and 1 patient T4a) had good scores (KPS $\geq 70$, ASA score 2). Subtotal resection was feasible for one patient with T4a tumor and partial resection was done for the patient with T4b tumor.

While patients younger than 60 years $(n=9,56.25 \%) ; 3$ patients ( 2 patients T4b and 1 patients T4a) had poor scores (KPS $\leq 70$, ASA score 3, 4); in all of them intracapsular debulking of the tumor for brainstem decompression was performed to improve the clinical manifestations of the patients. In 2 cases of them postoperative shunt was performed. One of them (grade T4b) the shunt was inserted 3 days after the surgery and in the second case (grade T4a); the

Table 4. ASA physical status classification system.

\begin{tabular}{cc}
\hline ASA score & Physical status \\
1 & A normal healthy patient \\
2 & A patient with a mild systemic disease \\
3 & A patient with an incapacitating systemic disease that is a constant threat to live \\
4 & A moribund patient not expected to survive 24 hours with or without operation \\
5 &
\end{tabular}


shunt was inserted 2 months after surgery because the patient developed hydrocephalus which was not apparent by the time of the surgery. In the other 6 patients (4 patients T4b and 2 patients T4a); gross total resection was achieved in 2 cases (one T4a and one T4b), subtotal resection was achieved in 4 cases ( 3 patients T4b and 1 patients T4a). Preoperative shunt for the hydrocephalus was performed in the all the 3 cases of $\mathrm{T} 4 \mathrm{~b}$.

Extent of resection was evaluated by post-contrast MRI brain done 3 months after surgery. Gross total resection was achieved in 2 cases (12.5\%). One of them was (grade T4a) and total resection was achieved without any intraoperative or postoperative sequale. In the other case preoperative shunt was performed followed by surgery with total resection of the tumor but after surgery the patient showed progression of her brainstem manifestation and the bulbar symptoms necessitating long-term ICU admission (one month) then gradual improvement of her manifestation over the next 3 months (Figure 1). Subtotal resection was achieved in 5 cases (31.25\%); two patients with tumor of grade T4a and three patients with tumor of grade T4b. Partial resection was achieved in 9 cases (56.25\%); six patients with tumor of grade T4b and three patients with tumor grade T4a. The surgical procedure was aborted in 3 cases due to severe intraoperative bleeding and in one of them; ventriculoperitoneal (VP) shunt was inserted the next day after surgery because of small hematoma in the tumor bed and blood clots the subarachnoid space causing delayed recovery in the patient conscious level after surgery.

Seven patients had postoperative brainstem manifestation. 3 of them showed progression of their preoperative brainstem dysfunction; 2 of them improved gradually over 3 months after surgery and 1 of them remained stationary. 4 cases developed new onset brainstem manifestation after surgery which had recovered in 3 of them.

Out of the 9 patients with preoperative bulbar manifestation 3 cases showed
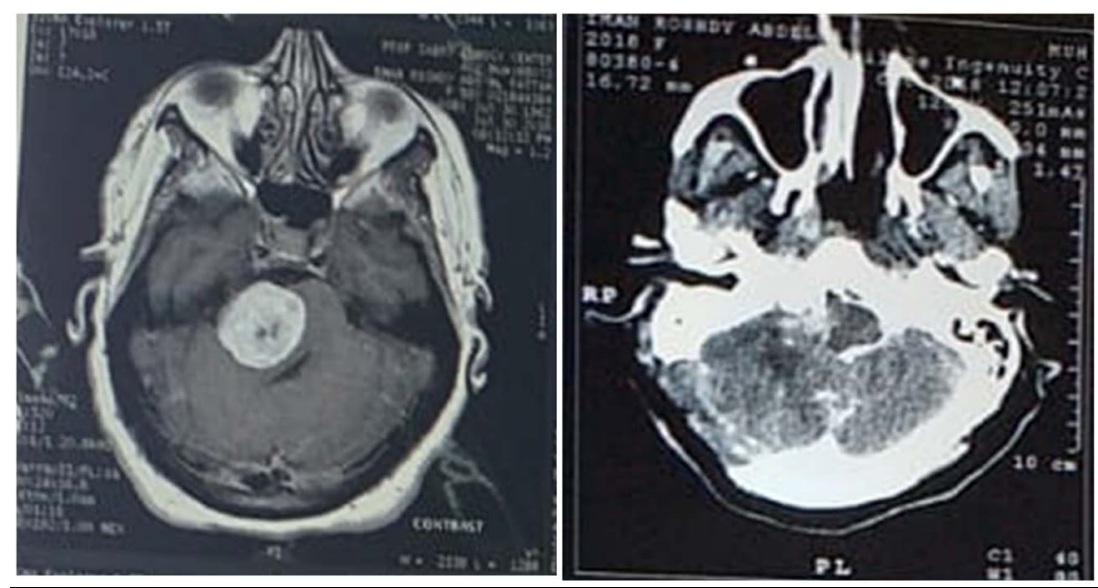

Figure 1. (a) T1-weighted gadolinium-enhanced axial MRI of a 53-year-old female showing a $4.9-\mathrm{cm}^{3}$ solid and cystic vestibular schwannoma with significant brain stem compression. (b) Follow-up computed tomography showing small hematoma in tumor bed and gross total resection of the tumor. 
improvement of their preoperative bulbar manifestation, 4 patients remained stationary and 2 patients showed worsening of their bulbar manifestation, we had 2 cases of preoperative tracheostomy in one of them the tracheostomy was removed 1 month after surgery and 3 cases had transient new onset bulbar manifestation that recovered gradually after surgery. We had 3 cases of postoperative tracheostomy; 2 cases with worsening bulbar manifestation and one case of newly developed bulbar manifestation, and one case needed jujenostomy due to severe bulbar manifestation and inability to swallow. We had 4 cases of mortality (25\%): 2 of them died from severe brainstem dysfunction (one of them had preoperative tracheostomy for severe associated bulbar manifestation). 1 case died from chest infection due to aspiration pneumonia due to severe bulbar manifestation and 1 case died from pulmonary embolism caused by deep venous thrombosis (DVT) due to long ICU stay.

Five patients with bad preoperative KPS and ASA scores needed long standing ICU stay (more than 1 month) because of their bad preoperative condition and not related to the surgical sequale. 4 patients with good preoperative KPS and ASA scores needed long standing ICU stay due to operative and postoperative sequales.

\section{Discussion}

Surgical management of VS has been evolved dramatically during the last century due to the progressive learning curve and the cumulative experience from the great achievements by the leading pioneers of neurosurgery and advancement in the surgical technical facilities. The first contribution from the legend Cushing was the reduction of mortality rates from $50 \%$ to $11 \%$. Walter Dandy reported a series of 5 patients with gross total resection without mortality. The aim of surgery for VS has been changed from just achieving a total resection without mortality to preserve the neurological function with achievement a good quality of life. This achievement is facilitated by advancement in the modern neurosurgical techniques and neuro-anaesthesia and availability of intraoperative neurophysiological monitoring for the brainstem and cranial nerves function [18] [19] [20] [21].

Patients with VS compressing the brainstem have variable clinical presentation including multiple cranial nerve dysfunction, brainstem compression signs or intracranial hypertension due to hydrocephalus. Our study is focusing on the VS exerting variable degrees of brainstem compression. Although, the VS size is an important issue in determination of the clinical manifestation of VS and the degree of brainstem dysfunction but there is many reports that VS can compress the brainstem regardless its size. This can be explained by evident individual anatomical variation in the posterior fossa including osseous anatomy, size of the cerebellopontine cistern or even cerebellar atrophy. Another important factor is the direction of tumor extension into CPA which influence the clinical presentation regardless of the size of the VS which is clearly differentiated and 
classified by Hannover grading scale rather than the Koos grading system which stress only on the tumor size [22]-[27].

Although, advancement in the modern neuroradiological tools and increased patient awareness that facilitated early diagnosis of VS without significant clinical manifestation. However, in developing countries lack of advanced healthcare system many cases of VS diagnosed late with advanced clinical presentation including branistem manifestation and significant bulbar manifestation, this late presentation made those patient more challenging for anaesthesia and surgical intervention which greatly affect the outcome [28] [29] [30]. In this study, we stressed on those patients coming late in their clinical presentation and we discussed our clinical and surgical challenges to stress on the value of early diagnosis and management to prevent many sequales that could be avoided with early diagnosis and management.

In our study of 16 patients with VS compressing the brainstem; manifestation of brainstem compression was recorded in $43.75 \%$ of patients, significant bulbar manifestation occurred in $56.25 \%$ of patients and hydrocephalic manifestation in $62.5 \%$ of patients. These clinical manifestations were worse affecting the life-style and performance of those patients and were associated with significant morbidity that influenced our management plan for anaesthesia and surgery. Utilizing a scoring system for the patient performance and anaesthetic fitness was of a great paramount for our decision making [17].

The KPS and ASA score for anaesthesia were both utilized for preoperative assessment and management plan. We had 8 cases of good preoperative KPS and ASA score and in those cases we achieved total and subtotal resection of the tumor in $87.5 \%$ of cases while partial resection was done in $12.5 \%$ of cases; the reported incidence of newly diagnosed VS in many studies ranged from $86 \%$ to 97.9\% based on the tumor size, extension and patient clinical presentation [31] [32] [33] [34]. In the other 8 patients with bad preoperative KPS and ASA score we achieved only partial resection of the tumor without attempting to do total resection.

There was association between the degree of brainstem compression and the preoperative KPS and ASA score. In 5 patients with grade T4b there was a bad preoperative KPS and ASA score and 2 of them necessitated postoperative insertion of VP shunt due to progressive hydrocephalus. On the other hand, preoperative insertion of VP shunt had a positive effect of the preoperative scoring system. In 3 cases of grade T4b tumors; VP shunt was inserted before surgery which improved the KPS and anesthetic score for those patients and we are capable to achieved a subtotal resection in 2 patients and gross total resection in 1 patient and this is in favor of treating hydrocephalus in those patients to improve the surgical outcome and minimized the surgery related sequale.

Many studies showed no different in the outcome and morbidities in cases of VS with hydrocephalus either treated with direct surgery or shunt insertion prior to surgery. However, these studied advocated perioperative external ven- 
tricular drain increased the safety and decreased the surgery related morbidities. Nevertheless, in situations of long waiting list for the patients; leaving the patients with hydrocephalic manifestations untreated may cause deterioration of the clinical manifestations with increased negative influence on the surgical outcome and external ventricular drain insertion for those cases is associated with higher morbidity due to long standing external ventricular drain [8] [35].

In general; lines of management of VS generally include conservative follow up, microsurgical excision, and radiosurgery. However, surgery is the main treatment for VS with brainstem compression as it has severe life-threatening complications caused by more tumor growth. In cases of VS with brainstem compression; radiosurgery is not preferred as a treatment option as radiation induce tumor swelling and may cause deterioration of brainstem dysfunction. The main line of treatment for patients with VS with brainstem compression and neurological deterioration is tumor microsurgical excision with brainstem decompression and relief of intracranial hypertension caused by hydrocephalus [2] [31] [32].

Surgical resection of VS usually done through three main surgical approaches; the retrosigmoid, translabyrinthine, and middle fossa approaches. The middle fossa approach is unsuitable for VS with brainstem compression as it offers a very restricted path to the CPA. However, the retrosigmoid and translabyrinthine approaches permit surgical removal of any size of VS [9] [24] [36] [37] [38] [39] [40]. All our patients were operated upon through retrosigmoid approach. Lumbar drain was intra-operatively inserted in all cases except for 3 cases in which preoperative shunt was done. We drained 15 to $20 \mathrm{cc}$ cerebrospinal fluid (CSF) before dural opening to relax the cerebellum and facilitate tumor resection. Lateral part of the cerebellum was excised in 4 cases to facilitate the tumor dissection and exposure to avoid excessive cerebellar retraction and swelling. The surgical procedure was aborted in 3 patients due to acute cerebellar swelling and occurrence of intraoperative significant bleeding during attempting tumor exposure and dissection; in one of them VP shunt was inserted in the next day after surgery due to leading in the tumor bed and subarachnoid space.

Long ICU stay (more than 1 month) was recorded in 9 cases; out of them 5 patients had bad preoperative KPS and ASA scores and despite non aggressive surgical manipulation but the patient clinical manifestation was not in favor of discharging them outside the ICU because of the sequale of bulbar and brainstem manifestations. And 4 cases with good preoperative KPS and ASA scores developed surgically related sequale regarding brainstem dysfunction and bulbar manifestation that indicated long ICU stay. 5 of the cases with long ICU stay had tracheostomy ( 2 preoperatively and 3 postoperatively) and one patient necessitated jujenostomy for feeding. Many studied reported incidence of either long ICU stay or repeated admission of patients with high-risk VS in the first month postoperative due to surgery related sequale including brainstem, bulbar, hydrocephalic manifestations and surgical bed hematoma [32] [34]. 


\section{Limitation to This Study}

There are some limitations to this study including the limited number of cases in this study, about half of the cases presented with poor KPS and ASA scores which greatly affected our surgical plan and patient outcome and this study had not included previously treated cases either with surgery alone or with surgery and postoperative radiotherapy including Gamma Knife radiosurgery which may add more to the surgical challenge and outcome.

\section{Conclusion}

Vestibular schwannomas compressing the brainstem with or without hydrocephalus are a big challenge for neurosurgical practice. There is a high incidence of operative and postoperative adverse sequale. The outcome of patients treated with hydrocephalus prior to VS surgery is better than those who did initial VS surgery. Patients presented with significant brainstem, bulbar manifestation had worse outcome and needed longer ICU stay and longer time for recovery.

\section{Financial Support and Sponsorship}

None.

\section{Conflicts of Interest}

The authors declare no conflicts of interest regarding the publication of this paper.

\section{References}

[1] Matthies, C., Samii, M. and Krebs, S. (1997) Management of Vestibular Schwannomas (Acoustic Neuromas): Radiological Features in 202 Cases-Their Value for Diagnosis and Their Predictive Importance. Neurosurgery, 40, 469-481. https://doi.org/10.1227/00006123-199703000-00009

[2] Park, C.K., Jung, H.W., Kim, J.E., et al. (2006) Therapeutic Strategy for Large Vestibular Schwannomas. Journal of Neuro-Oncology, 77, 167-171. https://doi.org/10.1007/s11060-005-9015-y

[3] Tos, M., Stangerup, S.E., Cayé-Thomasen, P., et al. (2004) What Is the Real Incidence of Vestibular Schwannoma? Archives of Otolaryngology-Head and Neck Surgery, 130, 216-220. https://doi.org/10.1001/archotol.130.2.216

[4] Raslan, A.M., Liu, J.K., McMenomey Samii, M., et al. (2010) Functional Outcome after Complete Surgical Removal of Giant Vestibular Schwannomas. Journal of Neurosurgery, 112, 860-867. https://doi.org/10.3171/2009.7.JNS0989

[5] Mehrotra, N., Behari, S., Pal, L., et al. (2008) Giant Vestibular Schwannomas: Focusing on the Differences between the Solid and the Cystic Variants. British Journal of Neurosurgery, 22, 550-556. https://doi.org/10.1080/02688690802159031

[6] Pai, I., Bowman, J., Thomas, N., et al. (2011) Management of Large and Giant Vestibular Schwannomas. Skull Base, 21, 379-384.

https://doi.org/10.1055/s-0031-1287680

[7] Bambakidis, N.C., Lo, S.S. and Selman, W.R. (2011) Large Vestibular Schwannomas. Journal of Neurosurgery, 115, 894-895. 
https://doi.org/10.3171/2011.5.JNS11765

[8] Prabhuraj, A.R., Sadashiva, N., Kumar, S., et al. (2017) Hydrocephalus Associated with Large Vestibular Schwannoma: Management Options and Factors Predicting Requirement of Cerebrospinal Fluid Diversion after Primary Surgery. Journal of Neurosciences in Rural Practice, 8, 27-32.

[9] Matthies, C. and Samii, M. (1997) Management of 1000 Vestibular Schwannomas (Acoustic Neuromas): Clinical Presentation. Neurosurgery, 40, 1-9. https://doi.org/10.1097/00006123-199701000-00001

[10] Bandlish, D., Biswas, N. and Deb, S. (2014) Staging in Giant Vestibular Schwannoma Surgery: A Two Consecutive Day Technique for Complete Resection in Basic Neurosurgical Setups. Journal of Neurosciences in Rural Practice, 5, 225-230. https://doi.org/10.4103/0976-3147.133560

[11] Anderson, D.E., Leonetti, J., Wind, J.J., et al. (2005) Resection of Large Vestibular Schwannomas: Facial Nerve Preservation in the Context of Surgical Approach and Patient-Assessed Outcome. Journal of Neurosurgery, 102, 643-649. https://doi.org/10.3171/jns.2005.102.4.0643

[12] Gharabaghi, A., Samii, A., Koerbel, A., et al. (2007) Preservation of Function in Vestibular Schwannoma Surgery. Neurosurgery, 60, 124-127. https://doi.org/10.1227/01.NEU.0000249245.10182.0D

[13] Ansari, S.F., Terry, C. and Cohen-Gadol, A.A. (2012) Surgery for Vestibular Schwannomas: A Systematic Review of Complications by Approach. Neurosurgical Focus, 33, E14. https://doi.org/10.3171/2012.6.FOCUS12163

[14] Anaizi, A.N., Gantwerker, E.A., Pensak, M.L., et al. (2014) Facial Nerve Preservation Surgery for Koos Grade 3 and 4 Vestibular Schwannomas. Neurosurgery, 75, 671-675. https://doi.org/10.1227/NEU.0000000000000547

[15] Monfared, A., Corrales, E., Theodosopoulos, P., et al. (2016) Facial Nerve Outcome and Tumor Control Rate as a Function of Degree of Resection in Treatment of Large Acoustic Neuromas: Preliminary Report of the Acoustic Neuroma Subtotal Resection Study. Neurosurgery, 79, 194-203. https://doi.org/10.1227/NEU.0000000000001162

[16] World Health Organization (1980) World Health Organization International Classification of Impairments, Disabilities and Handicap. Geneva.

[17] Knuf, K.M., Maani, C.V. and Cummings, A.K. (2018) Clinical Agreement in the American Society of Anesthesiologists Physical Status Classification. Perioperative Medicine, 7, 14. https://doi.org/10.1186/s13741-018-0094-7

[18] Jian, B.J., Sughrue, M.E., Kaur, R., et al. (2011) Implications of Cystic Features in Vestibular Schwannomas of Patients Undergoing Microsurgical Resection. Neurosurgery, 68, 874-880. https://doi.org/10.1227/NEU.0b013e318208f614

[19] Iwai, Y., Ishibashi, K., Watanabe, Y., et al. (2015) Functional Preservation after Planned Partial Resection Followed by Gamma Knife Radiosurgery for Large Vestibular Schwannomas. World Neurosurgery, 84, 292-300. https://doi.org/10.1016/j.wneu.2015.03.012

[20] Samii, M., Gerganov, V. and Samii, A. (2006) Improved Preservation of Hearing and Facial Nerve Function in Vestibular Schwannoma Surgery via the Retrosigmoid Approach in a Series of 200 Patients. Journal of Neurosurgery, 105, 527-535. https://doi.org/10.3171/jns.2006.105.4.527

[21] Vijendra, K.J., Naveen, M., Rabi, N.S., et al. (2005) Surgery of Vestibular Schwannomas: An Institutional Experience. Neurology India, 53, 41-45.

https://doi.org/10.4103/0028-3886.15052 
[22] Rhoton, A.L. (2000) The Cerebellopontine Angle and Posterior Fossa Cranial Nerves by the Retrosigmoid Approach. Neurosurgery, 47, S93-S129. https://doi.org/10.1093/neurosurgery/47.3.S93

[23] Patni, A.H. and Kartush, J.M. (2005) Staged Resection of Large Acoustic Neuromas. Otolaryngology—Head and Neck Surgery, 132, 11-19.

https://doi.org/10.1016/j.otohns.2004.09.094

[24] Ramina, R., Coelho Neto, M., et al. (2007) Treatment of Large and Giant Residual and Recurrent Vestibular Schwannomas. Skull Base, 17, 109-117. https://doi.org/10.1055/s-2006-953510

[25] Silva, J., Cerejo, A., Duarte, F., et al. (2012) Surgical Removal of Giant Acoustic Neuromas. World Neurosurgery, 77, 731-735. https://doi.org/10.1016/j.wneu.2011.08.019

[26] Kumar, B., Behari, S., Jaiswal, A.K., et al. (2013) Large and Giant Vestibular Schwannomas: Does Cisterna Magna Hyperproteinorrhachia Influence Visual Status? Acta Neurochirurgica, 155, 63-69. https://doi.org/10.1007/s00701-012-1529-4

[27] Atlas, M.D., Perez de Tagle, J.R., Cook, J.A., et al. (1996) Evolution of the Management of Hydrocephalus Associated with Acoustic Neuroma. Laryngoscope, 106, 204-206. https://doi.org/10.1097/00005537-199602000-00018

[28] Nonaka, Y., Fukushima, T., Watanabe, K., et al. (2013) Contemporary Surgical Management of Vestibular Schwannomas: Analysis of Complications and Lessons Learned over the Past Decade. Neurosurgery, 72, 115. https://doi.org/10.1227/NEU.0b013e3182752b05

[29] Raftopoulos, C., Abu Serieh, B., Duprez, T., et al. (2005) Microsurgical Results with Large Vestibular Schwannomas with Preservation of Facial and Cochlear Nerve Function as the Primary Aim. Acta Neurochirurgica, 147, 697-706. https://doi.org/10.1007/s00701-005-0544-0

[30] Turel, M.K., D’Souza, W.P., Chacko, A.G. and Rajshekhar, V. (2016) Giant Vestibular Schwannomas: Surgical Nuances Influencing Outcome in 179 Patients. Neurology India, 64, 478-484. https://doi.org/10.4103/0028-3886.181558

[31] Gerganov, V.M. and Samii, M. (2012) Giant Vestibular Schwannomas. World Neurosurgery, 77, 627-628. https://doi.org/10.1016/j.wneu.2011.10.008

[32] Turel, M.K., Thakar, S. and Rajshekhar, V. (2015) Quality of Life Following Surgery for Large and Giant Vestibular Schwannomas: A Prospective Study. Journal of Neurosurgery, 122, 303-311. https://doi.org/10.3171/2014.10.JNS14534

[33] Harati, A., Scheufler, K.M., Schultheiss, R., et al. (2017) Clinical Features, Microsurgical Treatment, and Outcome of Vestibular Schwannoma with Brainstem Compression. Surgical Neurology International, 8, 45. https://doi.org/10.4103/sni.sni_129_16

[34] Hatch, J., Bauschard, M., Nguyen, S., et al. (2018) National Trends in Vestibular Schwannoma Surgery: Influence of Patient Characteristics on Outcomes. Otolaryngology_Head and Neck Surgery, 159, 102-109. https://doi.org/10.1177/0194599818765717

[35] Briggs, R.J., Shelton, C., Kwartler, J.A., et al. (1993) Management of Hydrocephalus Resulting from Acoustic Neuromas. Otolaryngology_Head and Neck Surgery, 109, 1020-1024. https://doi.org/10.1177/019459989310900608

[36] Ramamurthi, B. (1990) Acoustic Neurinomas in Developing Countries. International Symposium on Acoustic Neurinomas. India Neurology, 38, 223.

[37] Van de Langenberg, R., Hanssens, P.E.J., van Overbeeke, J.J., et al. (2011) Manage- 
ment of Large Vestibular Schwannoma. Part I. Planned Subtotal Resection Followed by Gamma Knife Surgery: Radiological and Clinical Aspects. Journal of Neurosurgery, 115, 875-884. https://doi.org/10.3171/2011.6.JNS101958

[38] Van de Langenberg, R., Hanssens, P.E.J., Verheul, J.B., et al. (2011) Management of Large Vestibular Schwannoma. Part II. Primary Gamma Knife Surgery: Radiological and Clinical Aspects. Journal of Neurosurgery, 115, 885-893. https://doi.org/10.3171/2011.6.JNS101963

[39] Zhang, Z., Wang, Z., Huang, Q., et al. (2012) Removal of Large or Giant Sporadic Vestibular Schwannomas via Translabyrinthine Approach: A Report of 115 Cases. ORL: Journal for Oto-Rhino-Laryngology and Its Related Specialties, 74, 271-277. https://doi.org/10.1159/000343791

[40] Mamikoglu, B., Weit, R.J. and Esquivel, C.R. (2002) Translabrynthine Approach for the Management of Large and Giant Vestibular Schwannomas. Otology \& Neurotology, 23, 224-227. https://doi.org/10.1097/00129492-200203000-00020

\section{Abbreviations}

KPS $=$ Karnofsky performance score,

ASA = American Society of Anaestheiologist,

$\mathrm{VS}=$ Vestibular schwannomas,

$\mathrm{CPA}=$ Cerebellopontine angle,

$\mathrm{H}-\mathrm{B}=$ House-Brackmann,

$\mathrm{CT}=$ Computed tomography,

MRI = Magnetic resonance imaging. 